Abstract P4 Table 1

\begin{tabular}{|c|c|c|c|c|c|c|}
\hline \multirow[t]{2}{*}{ Pleural Procedure } & \multirow{2}{*}{$\begin{array}{l}\text { Total Performed over } \\
\text { Study Period }\end{array}$} & \multicolumn{2}{|c|}{ Intercostal Artery (ICA) Identification } & \multicolumn{3}{|c|}{ Cases where ICA Identification Altered Site Selection } \\
\hline & & $\begin{array}{l}\text { Attempt to Identify \& } \\
\text { (\% of total performed) }\end{array}$ & $\begin{array}{c}\text { Successfully Identified \& } \\
\text { (\% of Attempted Identification) }\end{array}$ & $\begin{array}{c}\text { Number of } \\
\text { Cases }\end{array}$ & $\begin{array}{c}\text { (\% of ICA Cases } \\
\text { Successfully Identified) }\end{array}$ & $\begin{array}{l}\text { (\% of all procedures } \\
\text { performed) }\end{array}$ \\
\hline Medical Thoracoscopy & 74 & $71 \quad(95.9)$ & $25 \quad(35.2)$ & 7 & $(28.0)$ & (9.5) \\
\hline Image Guided Pleural Biopsies & 47 & $\begin{array}{ll}46 & (97.9) \\
\end{array}$ & $32 \quad(69.6)$ & 16 & $(50.0)$ & $(34.0)$ \\
\hline Intercostal Chest Drain & 43 & $38 \quad(88.4)$ & $20 \quad(52.6)$ & 6 & $(30.0)$ & $(14.0)$ \\
\hline Indwelling Pleural Catheter & 33 & $32 \quad(97.0)$ & $11 \quad(34.4)$ & 2 & $(18.2)$ & $(6.1)$ \\
\hline Therapeutic Aspiration & 166 & $159 \quad(95.8)$ & $80 \quad(50.3)$ & 19 & $(23.8)$ & (11.4) \\
\hline Diagnostic Aspiration & 41 & $\begin{array}{ll}40 & (97.6) \\
\end{array}$ & $\begin{array}{ll}24 & (60.0) \\
\end{array}$ & 6 & $(25.0)$ & $(14.6)$ \\
\hline All procedures & 404 & $\begin{array}{ll}386 & (95.5)\end{array}$ & $\begin{array}{ll}192 & (49.7)\end{array}$ & 56 & (29.2) & (13.9) \\
\hline
\end{tabular}

it remains important to choose intervention sites to avoid the intercostal arteries (ICA). Previous data suggest that the ICA can follow a tortuous course especially in the elderly. The use of colour Doppler to identify intercostal and collateral arteries has been shown to be accurate in research studies and may assist in selecting a safe intervention site.

This study aimed to prospectively assess identification of the ICA in routine practice and the effect on procedure site selection. Methods Data on identification of the ICA was prospectively collected as part of routine clinical care and documented in the pleural procedure records in a tertiary centre between July 2015 and July 2016. Successful identification of the ICA and its influence in choosing the procedure site was recorded.

Results 404 procedures were carried out over the study period. The mean age of the patients was 69.3 years (sd 14.2).

Identification of the ICA was attempted in 386 (95.5\%) procedures and the ICA was identified within the intercostal space in $192(49.7 \%)$ of cases.

The site of the procedure was altered after ICA detection in $56 / 192(29.2 \%)$ of procedures and in 16/32 (50.0\%) of image guided pleural biopsies.

In $7 / 192(3.6 \%)$ procedures the ICA was identified in all rib spaces at potential intervention sites, leading to the procedure not being attempted. No complications related to post procedure haemorrhage were reported.

A more detailed analysis of the identification of the ICA and its influence on practice by procedure type is shown in Table 1.

Conclusion Screening for the ICA in routine clinical practice influences procedural site selection.

In some cases identification can result in abandoning a procedure, which may have led to intercostal bleeding. Patient position and potential rib crowding may explain differences in the rates of successful identification between procedures.

If these findings are replicated in larger prospective studies, identification of the ICA may become routine practice to maximise safety.

\section{P5 BLOODY EFFUSIONS: DO THE PATIENT'S CLOTTING RESULTS OR ANTITHROMBOTIC MEDICATIONS MATTER?}

L Brockbank, R Pinto, M Gautam. Royal Liverpool and Broadgreen University Hospitals NHS Trust, Liverpool, UK

\subsection{6/thoraxjnl-2016-209333.148}

Introduction and objectives Current British Thoracic Society Guidelines state that a non-urgent pleural aspiration should be delayed in anti-coagulated patients until the international normalised ratio (INR) is $<1.5{ }^{1}$ There is no specific guidance around the use of antiplatelet/other medications, which may further increase bleeding risk. This creates dilemma for clinicians regarding the best timing of performing pleural procedures to reduce bleeding risk. This study reviewed the potential impact of abnormal clotting results and/or anti-thrombotic medications on the occurrence of blood-stained pleural fluid at aspiration.

Methods This was a retrospective study, in a large teaching hospital, of all pleural procedures performed between 2013-15, where potential bleeding risk data was available. Diagnostic and therapeutic aspirations as well as intercostal chest drain insertions performed by the Respiratory team were included. An antithrombotic medication score for prescribed medications was recorded as follows: aspirin/prophylactic low molecular weight heparin $(\mathrm{LMWH})=1$, clopidogrel/ticagrelor $=2$, warfarin/therapeutic $\mathrm{LMWH}=3$, in addition to pre-procedure INR, APTT and platelet count. Pleural effusions were divided into 'bloodstained' and 'non blood-stained' and the medication score, INR, APTT and platelet count compared between the two groups.

Results 207 cases were analysed: 73\% exudates, 27\% transudates. Pleural fluid cytology was available in $77 \%$ cases, of which $25 \%$ were malignant. The results for the total sample size $(n=207)$ is shown in the attached table. For blood stained effusions, 33\% were malignant vs. non blood stained, $17 \%$ malignant. Sub group analysis of malignant and benign effusions showed the same trend for blood stained vs. non blood stained effusions.

Abstract P5 Table 1 Comparison of pre-pleural procedure bleeding risk variables for blood stained versus non-blood stained pleural effusions

\begin{tabular}{llll}
\hline $\begin{array}{l}\text { Potential pre-procedure } \\
\text { bleeding risk variable }\end{array}$ & $\begin{array}{l}\text { blood- } \\
\text { stained } \\
(\mathbf{n}=60)\end{array}$ & $\begin{array}{l}\text { non-blood stained } \\
(\mathbf{n}=147)\end{array}$ & $\begin{array}{l}\text { statistical } \\
\text { significance }\end{array}$ \\
\hline $\begin{array}{l}\text { Mean anti-thrombotic medication } \\
\text { score }\end{array}$ & $1.21(0-4)$ & $1.19(0-4)$ & $\mathrm{p}=0.91$ \\
$\begin{array}{l}\text { Mean pre-procedure INR } \\
\text { Mean pre-procedure APTT }\end{array}$ & $\begin{array}{l}1.24(1-4.2) \\
30.74(19-\end{array}$ & $31.74(1-3)$ & $\mathrm{p}=0.63$ \\
& $93.8)$ & & $\mathrm{p}=0.50$ \\
Mean pre-procedure platelet & $341 \times 10^{9}$ & $293 \times 10^{9}(44-$ & $\mathrm{p}=0.06$ \\
count & $(72-614)$ & $1156)$ & \\
\hline
\end{tabular}

Conclusion Deranged coagulation or prescribed antithrombotics pre-pleural procedure do not appear to significantly increase the likelihood of a obtaining a blood-stained pleural effusion. The aetiology of blood stained pleural effusions is more likely multifactorial and should not always be attributed to a coagulation results or medication related increased bleeding risk. Further study could help determine how to better assess bleeding risk prior to pleural procedures. 


\section{REFERENCE}

1 Pleural procedures and thoracic ultrasound: BTS guideline 2010.

\section{\begin{tabular}{l|l} 
P6 & SIGNIFICANCE OF MINIMAL PLEURAL EFFUSION IN
\end{tabular} NON-SMALL CELL LUNG CANCER}

${ }^{1} \mathrm{GA}$ Martin, ${ }^{2} \mathrm{~S}$ Tsim, ${ }^{3} \mathrm{~J}$ MacLay, ${ }^{1} \mathrm{C}$ Stewart, ${ }^{1} \mathrm{KG}$ Blyth. 'Queen Elizabeth University Hospital, Glasgow, UK; ${ }^{2}$ Institute of Cancer Sciences, University of Glasgow, Glasgow, UK; ${ }^{3}$ Glasgow Royal Infirmary, Glasgow, UK

\subsection{6/thoraxjnl-2016-209333.149}

Introduction and objectives Recent publications report a significant survival disadvantage associated with minimal pleural effusion (MiniPE) at presentation of non-small cell lung cancer (NSCLC). MiniPE is defined when an effusion is too small for thoracentesis or where aspiration cytology is negative. Occult pleural metastases (OPM), indirect pathophysiology or comorbidity may cause MiniPE, but staging beyond thoracentesis is rarely performed. Assumption of OPM and therapeutic nihilism may contribute to poor outcomes. We assessed the prognostic impact of MiniPE in potentially radically-treatable NSCLC (Stage I-IIIA), oncologists' attitudes to treatment planning and the final treatment delivered.

Methods Electronic records and baseline imaging were reviewed retrospectively in 441 consecutive diagnoses of NSCLC made over 6 months in 2009. Stage I-IIIA patients were dichotomized into: No effusion and MiniPE. Malignant effusion (Stage IV) cases were recorded for comparison. The impact of effusion status on overall survival (OS) was estimated using Kaplan-Meier methodology. The probable cause of MiniPE was assessed indirectly using follow-up imaging/records. 3 Clinical Oncologists were surveyed for theoretical treatment plans in 8 randomlyselected MiniPE Stage I-IIIA cases based on anonymised imaging and history. These 24 plans were compared to the treatment delivered in MiniPE patients.

Results 103/441 (23\%) patients had MiniPE. 167/441 (38\%) were Stage I-IIIA; $26 / 167$ (16\%) of these had MiniPE. OS based on effusion status (Stage I-IIIA) is shown in Figure 1. 28/103 $(17 \%)$ MiniPE patients survived $<30$ days and had limited postdiagnosis imaging. These were excluded from probable cause analyses. Of the remaining 75/103, 20 (27\%) had radiological evidence of progressive pleural malignancy. Radical treatment was delivered in 4/26 (15\%) Stage I-IIIA MiniPE cases but advocated in $17 / 24(71 \%)$ theoretical plans, which showed significant inconsistencies.

Conclusions These retrospective data confirm the negative prognostic impact of MiniPE and suggest the prevalence of OPM is at least $27 \%$ in Stage I-IIIA NSCLC. This is likely an underestimate given our limited data in poor prognosis patients. Radical treatment was rarely delivered despite aggressive treatment plans. A prospective study utilising thoracoscopic staging could define the true prevalence of OPM in MiniPE. Objective staging might improve decision-making, radical treatment rates and OS in this context.

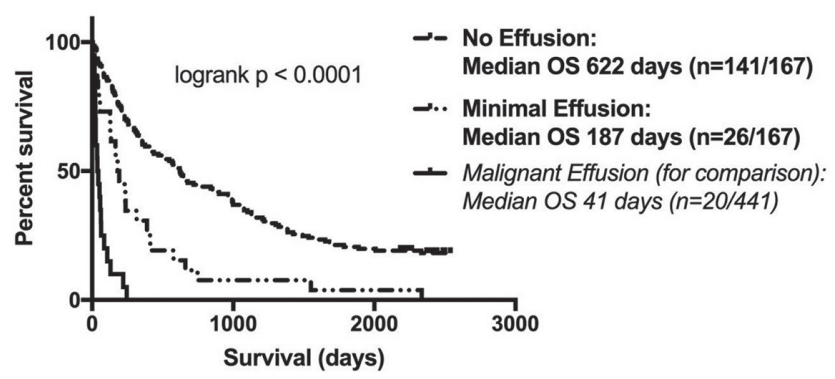

Abstract P6 Figure 1 Stage I-IIIA NSCLC Survival by Effusion Status

\section{P7 CLINICIANS' PERSPECTIVES OF HEALTH RELATED QUALITY OF LIFE AND PRIORITIES IN DECIDING MANAGEMENT FOR MALIGNANT PLEURAL EFFUSION}

${ }^{1} \mathrm{P}$ Sivakumar, ${ }^{1} \mathrm{D}$ Curley, ${ }^{2} \mathrm{NM}$ Rahman, ${ }^{3} \mathrm{YCG}$ Lee, ${ }^{4} \mathrm{D}$ Feller-Kopman, ${ }^{5} \mathrm{~A}$ West, ${ }^{5} \mathrm{~L}$ Ahmed. ${ }^{1}$ King's College London, London, UK; ${ }^{2}$ Oxford Centre for Respiratory Medicine, Oxford, UK; ${ }^{3}$ Institute for Respiratory Health and University of Western Australia, Perth, Australia; ${ }^{4}$ John Hopkins Hospital, Baltimore, USA; ${ }^{5}$ St Thomas' Hospital, London, UK

\subsection{6/thoraxjnl-2016-209333.150}

Introduction Malignant pleural effusion (MPE) management has dramatically changed in the last decade with the increasing use of indwelling pleural catheters (IPC) and thoracoscopy. Although treatment is aimed at improving health related quality of life (HRQOL), data on outcomes are limited, with management guided by clinician perspectives and experiences.

Aims We sought clinician perspectives of HRQOL for patients with MPE and its impact on decision making worldwide. We present the UK data.

Methods We invited all respiratory doctors in the UK to complete an online survey advertised in the British Thoracic Society newsletter and by e-mail. Responses to questions with ranked options were assigned consecutive integers with lower values indicating a more favoured or higher prioritised response. Responses to best answer questions are presented as frequencies and percentages.

Results 121 UK-based doctors (104 consultants, 1 associate specialist, 16 respiratory registrars) completed the survey.

Factors determining HRQOL (rank 1-9): shortness of breath and chest pain (mean rank 1.48) and functional status (mean rank 2.57) were ranked the most important. Social set up - mean rank 5.16, depression/anxiety - mean rank 5.22, tumour type and stage - mean rank 5.78, distance to travel for medical care mean rank 5.86, age - mean rank 6.59, financial difficulties from treatment - mean rank 8.27.

Factors in the decision to offer intervention for MPE (rank 16): breathlessness ranked highest (mean rank 1.83) followed by the risk of significant harm from procedure vs chance of benefit (mean rank 2.73).

Perspectives on which interventions most improve HRQOL are presented in Figure 1. 